\title{
Stability issues in 3D BEM formulations for transient elastodynamics
}

\author{
C. G. Panagiotopoulos ${ }^{1}$ \& G. D. Manolis ${ }^{2}$ \\ ${ }^{1}$ Department of Continuum Mechanics, University of Seville, Spain \\ ${ }^{2}$ Department of Civil Engineering, \\ Aristotle University of Thessaloniki, Greece
}

\begin{abstract}
Time-domain boundary element method formulations (TD-BEM) are quite versatile in reproducing the transient response of finite and semi-infinite solid media and offer a number of advantages compared to transformed domain (Fourier or Laplace) approaches. They are known, however, to be prone to numerical instability, especially after a very large number of time steps. This seems to be more of a problem with 1D as compared to 3D formulations, although it is present in the latter ones. A careful investigation of the problem reveals that if the conventional BEM formulation using displacement and traction variables is replaced with one using velocity and traction pairs, the problem is ameliorated to a large extent and much better accuracy results.
\end{abstract}

Keywords: elastodynamics, stability, time-marching schemes, reciprocal theorems, convolution integrals.

\section{Introduction}

Although the importance of TD-BEM formulations in studying wave motion problems in elastic continua is indisputable, the conventional formulation in terms of the displacement and traction vectors (DBEM) has a latent problem when a large number of time steps are required. More specifically, unstable behaviour may occur that is known as "intermittent instability" [1]. Various schemes have been proposed in recent years, many of them drawn from finite element methodologies (such as the Wilson-theta integration algorithm) to eliminating or reduce this unstable behaviour of the time convolution integrals associated with the transient BEM. All such methods basically seek to modify 
the underlying boundary integral equations so as to optimize the stability behaviour. Although stability may be enforced, it is usually accompanied by a reduction in accuracy, which is the consequence of the introduction of artificial damping and of natural period elongation [2]. Moreover, these algorithmic techniques are somewhat cumbersome, operating at different levels of complexity, and difficult to implement in existing BEM computer software codes. An alternative approach, as outlined in a recently by the present authors [3], is to introduce a new formulation based on the velocity reciprocal theorem (VBEM). This formulation has been successfully tested on simple problems, namely the single degree of freedom system and one dimensional wave propagation, with superior performance regarding stability as compared to formulations using the displacement reciprocal theorem. In this work, the fully 3D formulation is implemented following work outlined in ref. [4] and tested against benchmark problems. Again, overall superior performance of the VBEM compared to the DBEM is again established.

\section{Velocity reciprocal theorem for 3D elastodynamics}

Time domain BEM formulations use Graffi's reciprocal theorem [5], which employs displacement and traction fields and essentially is a work statement defined for a regular region $V$ with boundary $S$ (see Fig. 1) and material properties $p, c_{1}, c_{2}$ (mass density, pressure and shear wave speeds). In this work, we adopt an alternative strategy using a power statement that employs velocities in lieu of displacements. Specifically, consider two distinct elastodynamic states $\left[u_{i}, t_{i}, b_{i}\right]$ and $\left[u_{i}{ }^{\prime}, t_{i}{ }^{\prime}, b_{i}{ }^{\prime}\right]$ comprising displacements, tractions and body forces in $V$ with appropriate initial conditions. Next, define the velocity as the time derivative of displacement. For time $t \geq 0$ and by assuming (for simplicity) zero initial conditions, the following reciprocal theorem holds true

$$
\int_{S} t_{i} * v_{i}^{\prime} d S+\int_{V} \rho b_{i} * v_{i}^{\prime} d V=\int_{S} t_{i}^{\prime} * v_{i} d S+\int_{V} \rho b_{i}^{\prime} * v_{i} d V
$$

where operation * denotes Riemann's convolution. Utilizing this theorem with one state identified with the solution sought and the other with the fundamental singular solution pair, a velocity-based integral identity is:

$$
c_{i j}(\xi) v_{i}(\xi, t)=\int_{S}\left(\dot{G}_{i j} * t_{i}(x)-F_{i j} * v_{i}(x)\right) d S(x)+p \int_{V} \dot{G}_{i j} * b_{i}(x) d V(x)
$$

The fundamental velocity solution $\dot{G}_{i j}(x, t ; \xi, \tau=0)$ is computed by noting that

$$
\int_{c_{1}^{-1}}^{c_{2}^{-1}} \delta(t-\lambda r) \lambda d \lambda=\frac{t}{r^{2}}\left[H\left(t-\frac{r}{c_{1}}\right)-H\left(t-\frac{r}{c_{2}}\right)\right]
$$

and is given as: 


$$
\begin{aligned}
\dot{G}_{i j}=(4 \pi \rho)^{-1}\left\{a_{i j}(r) \dot{\delta}\left(t-\frac{r}{c_{1}}\right)+b_{i j}(r) \dot{\delta}\left(t-\frac{r}{c_{2}}\right)\right. & \\
& \left.+c_{i j}(r) \frac{t}{r^{2}}\left[\delta\left(t-\frac{r}{c_{1}}\right)-\delta\left(t-\frac{r}{c_{2}}\right)\right]+c_{i j}(r) \frac{1}{r^{2}}\left[H\left(t-\frac{r}{c_{1}}\right)-H\left(t-\frac{r}{c_{2}}\right)\right]\right\}
\end{aligned}
$$

where spatial coefficients $a_{i j}, b_{i j}, c_{i j}$ are the same as those appearing the conventional displacement BEM formulation [6] (i.e., the DBEM). This new formulation can easily be extended to incompressible materials [7], and becomes independent of the dilatational wave speed.

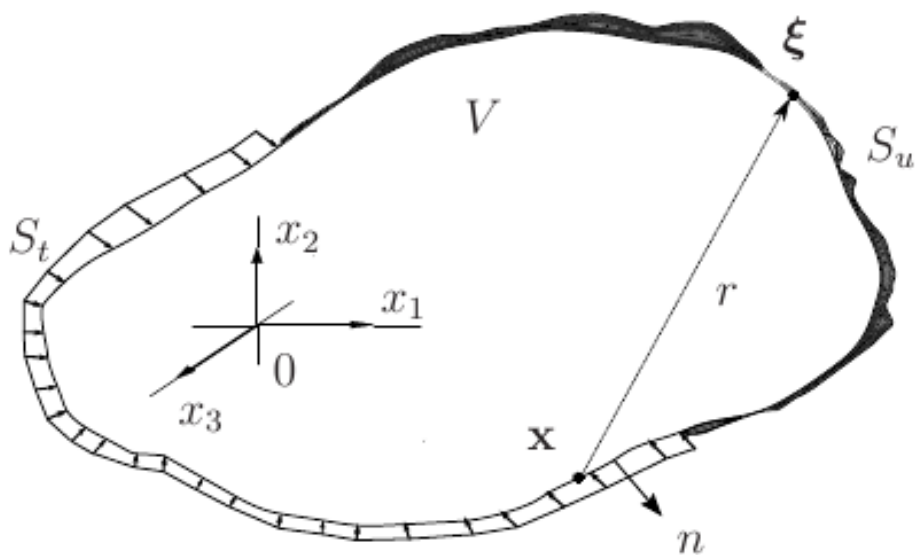

Figure 1: $\quad$ Elastic solid body and BEM notation.

\section{BEM formulations for displacements and for velocities}

In order to proceed with formulating a BEM algorithm for elastodynamics, we need to establish spatial as well as temporal discretizations of the field variables. First, to integrate the time convolutions analytically, we proceed by dividing the time interval $[0, t]$ into $N$ equal time steps of length $h$ and approximating any of the tractions, displacements (DBEM) or velocities (VBEM) in terms of basis functions. To study accuracy and stability, we consider a linear evolution of any time variable $\mathrm{f}(\mathrm{t})$ across a time-step as

$$
f(t)=\sum_{n=1}^{N} \phi_{n}(t)\left(\frac{t_{n}-t}{h} f_{n-1}+\frac{t-t_{n-1}}{h} f_{n}\right)
$$

where

$$
\phi_{n}(t)=H\left(t-t_{n-1}\right)-H\left(t-t_{n}\right)
$$

and $t_{n-}=n h, H(t)$ is the Heaviside function while $f_{n-1}, f_{n}$ are values at discrete times $t_{n-1}, t_{n}$, respectively. 
Regarding spatial discretization, a family of 2D surface isoparametric elements is used, namely the four, eight and nine node quadrilaterals (see Fig. 2). Spatial variation of any field variable $\mathrm{f}(\mathrm{x})$ is expressed in terms of the same shape functions $M_{a}$ used for the geometry, i.e.,

$$
f(x)=\sum_{a=1}^{q} M_{a}(x) f_{a}
$$

In the above, $q$ is the number of element's nodes, while $f_{a}$ is a nodal value in space. After replacement in the BEM statement, space-time interpolation products arise, which in compact form read as follows:

$$
f(x, t)=\sum_{n=1}^{N} \sum_{a=1}^{q} N_{1, n}(t) M_{a}(x) f_{a, n-1}+N_{2, n}(t) M_{a}(x) f_{a, n}
$$

In the above,

$$
N_{1, n(t)}=\phi_{n}(t) \frac{t_{n}-t}{h}, N_{2, n(t)}=\phi_{n}(t) \frac{t-t_{n-1}}{h}
$$

Analytical spatial integrations of the resulting BEM system matrix coefficients are not in general possible and therefore numerical quadrature is used. Fundamental solution-shape function products are approximated by application of the Gauss-Legendre quadrature formula. There are two basic cases of integrand behaviour, namely non-singular and singular. Regarding the latter, weak singularities in both DBEM and VBEM are done by element subdivisions,

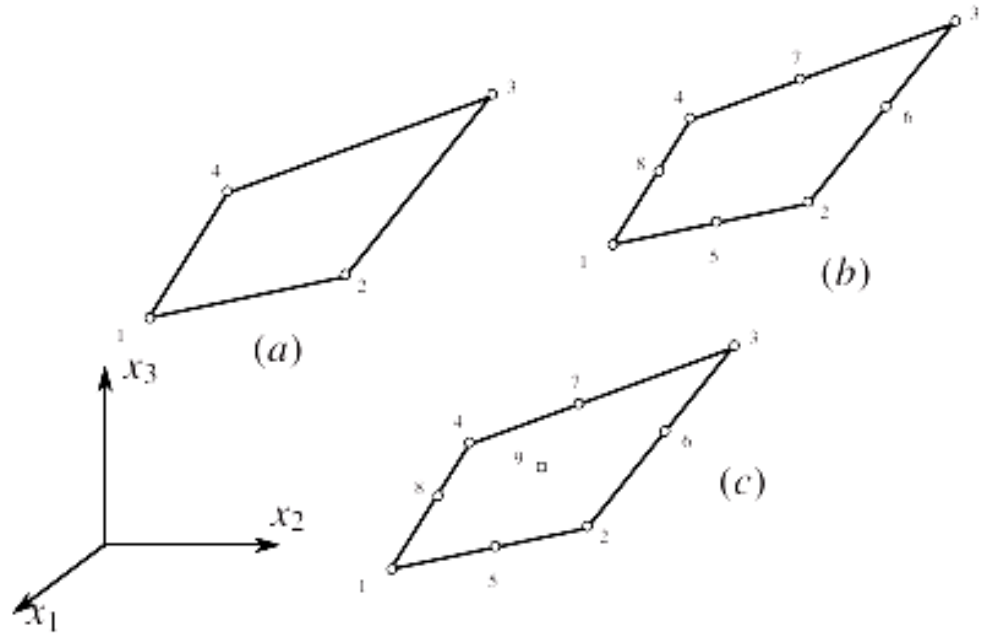

Figure 2: $\quad$ Surface boundary elements: (a) four, (b) eight and (c) nine nodes. 
followed by a series of transformations that reduces the order of singularity in the kernels by one and transforms them to regular ones. In the case of strongly singular traction kernels, the diagonal sub-matrices are computed utilizing indirect schemes based on the rigid body motion concept [8].

An important point for accuracy reasons in such formulations is identification of active zones of the integration along an element as it is sweep by the $\mathrm{P}$ and $\mathrm{S}$ waves. This is accomplished by invoking the causality principle [9] in the numerical integration scheme and employing element subdivision.

\section{Stability analysis and BEM performance}

We introduce a formal definition of stability as "Stability means that any 'initial' conditions at time $t$ given by errors in the response, which may be due to roundoff in the computer, do not grow in the integration". According to this definition [10] we distinguish between accuracy of a numerical method and its stability and focus on the latter. As example, we select a solid cube (see Fig. 3) with side length equal to $\mathrm{L}_{\text {tot }}$. We discretize each side into ne $\times$ ne square boundary elements of length $\mathrm{L}=\mathrm{L}_{\text {tot }} /$ ne. Next, we introduce the dimensionless CourantFredricks-Lewy (CFL) parameter as $\beta=\mathrm{cdt}_{\mathrm{W}}$, where $\mathrm{c}$ is either the $\mathrm{P}$ or $\mathrm{S}$ wave speed and $\mathrm{L}_{\mathrm{W}}$ a representative boundary element length, such as the minimum distance between two nodes. We adopt the $\mathrm{S}$ wave speed in the CFL measure, since it has the advantage of being applicable to the incompressible material case. Then we assign fixed kinematic (displacements and velocities) boundary conditions on the negative $\mathrm{x}_{1}$ face of the cube, zero tractions boundary conditions on the remaining faces and introduce unit initial 'error' for all boundary nodal values so as to trace its time evolution.

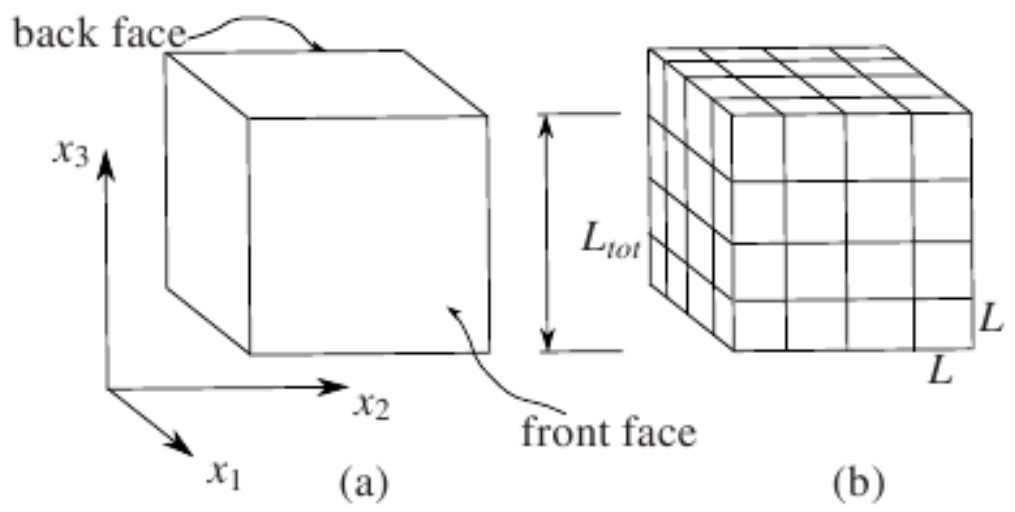

Figure 3: (a) Geometry of solid cube used to study stability (b) BEM discretization. 


\subsection{Stability aspects of the DBEM formulation}

The DBEM stability regions in terms on CFL parameter $\beta_{2}$ can be broken down in two parts: For $\beta_{2} \leq 0.6$, the time-stepping algorithm shows instability that becomes more noticeable for diminishing $\beta_{2}$. For values of $\beta_{2}>0.6$, we observe a stable performance since the initial 'error' is damped as time evolves. Meanwhile, if the 'error' evolution is tracked over for a long time, sudden instability occurs as shown in Fig. 4. This has been reported elsewhere [11], and if we further increase $\beta_{2}$, we find a small stability region between $1.1 \leq \beta_{2} \leq 1.2$, since for a large number of time steps the initial 'error' decreases. For values

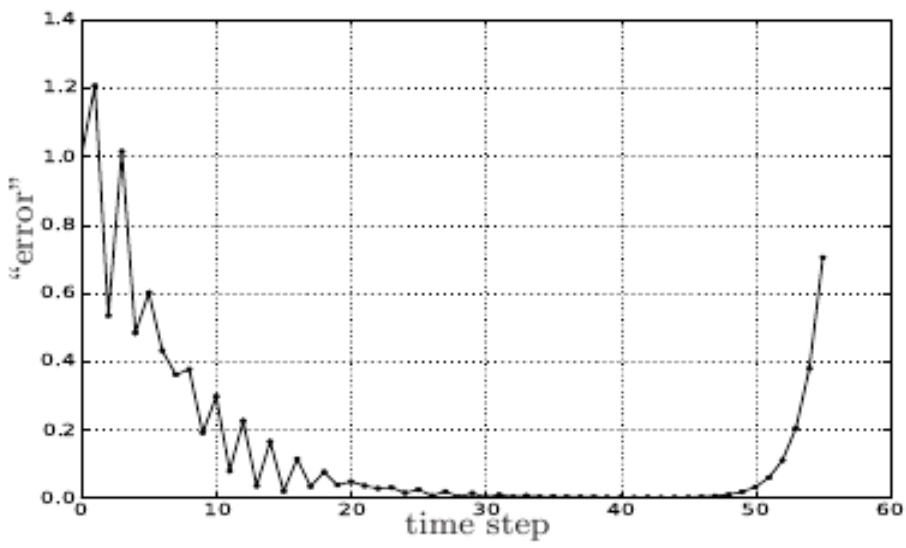

(a)

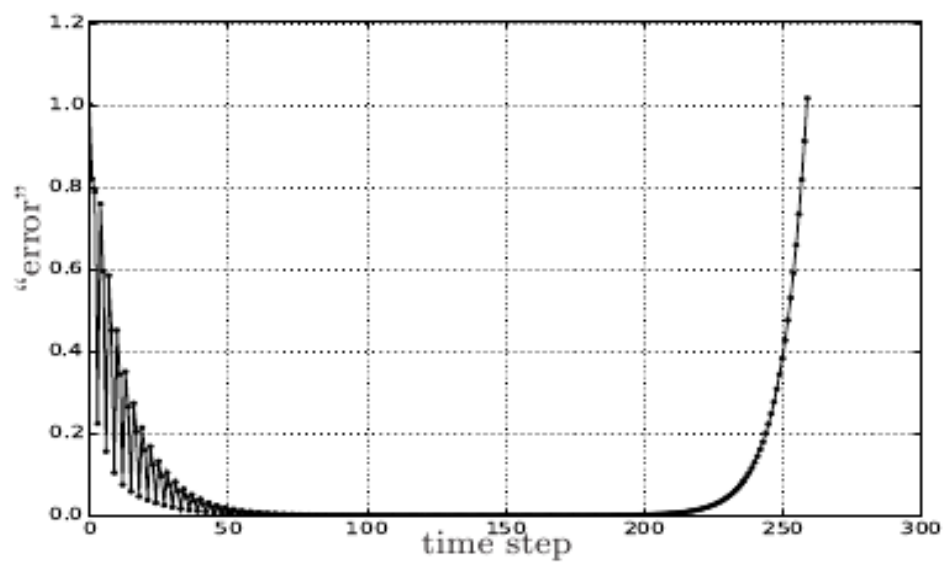

(b)

Figure 4: Time evolution of DBEM displacement 'error' (a) $\beta_{2}=0.8$ (b) $\beta_{2}=0.9$. 


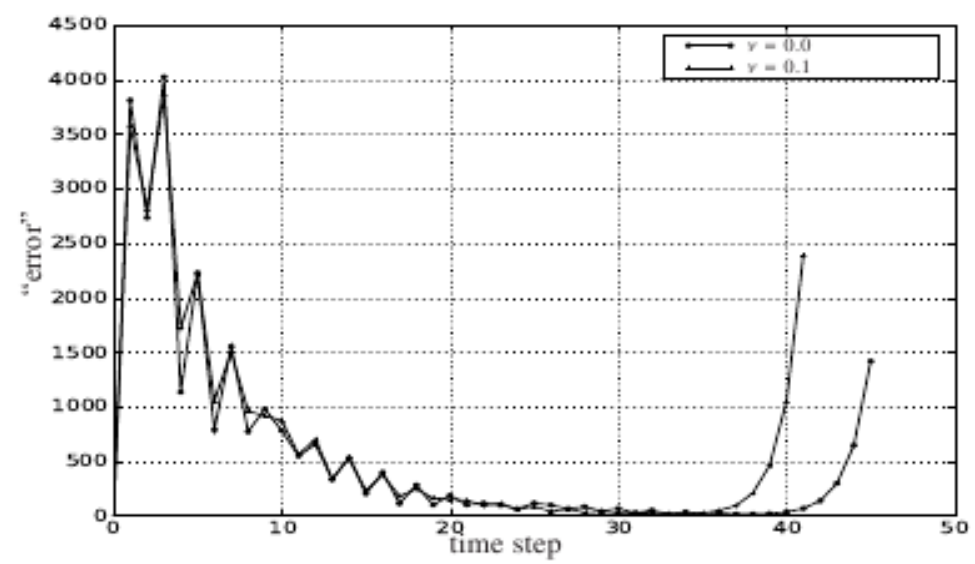

Figure 5: Time evolution of BDEM traction 'error' $\beta_{2}=0.75$ (a) $v=0$ (b) $v=0.1$.

greater than $\beta_{2}>1.2$, instability occurs once again. This kind of behavior, which is referred to in Ref. [12] as intermittent instability, is unacceptable as one cannot provide coherent guidelines on an appropriate choice of the time step.

Also, for Poisson's ratio values greater than zero, the DBEM present pronounced instability problems. This is observed in Fig. 5, where the 'error' evolution for tractions it is shown at a fixed $\beta_{2}$ value, but for two distinct values of Poisson's ratio $v$. For non-zero $v$, instability is manifested even for a small number of time steps. Furthermore, for large values of $v$ it was impossible to define any stability region regarding the $\beta_{2}$ parameter, in terms of long time evolution, since sudden instability occurred.

\subsection{Stability aspects of the VBEM formulation}

The VBEM stability performance is shown here to be smoother and more predictable. Most important, there exists a lower limit for $\beta_{2}$ beyond which the time-stepping algorithm is found to be stable. Specifically, for Poisson's ratio range $0 \leq v \leq 0.5$, the stability region was identified as $0.7 \leq \beta_{2} \leq 0.9$. These types of simulations are shown in Fig. 6. Corresponding values for the $\beta_{1}$ parameter may easily be retrieved, and we note that for large values of $v$, correspondingly very large values of $\beta_{1}$ result which produce substantial numerical damping. This unwelcome effect, however, may be reduced using with finer boundary element meshes.

In sum, the superior VBEM performance is probably due to the structure of the velocity fundamental solution, and specifically to the presence of the Dirac delta function that has a more 'local' space-time effect. In contrast, the displacement fundamental solution of the BDEM involves use of the Heaviside function. 


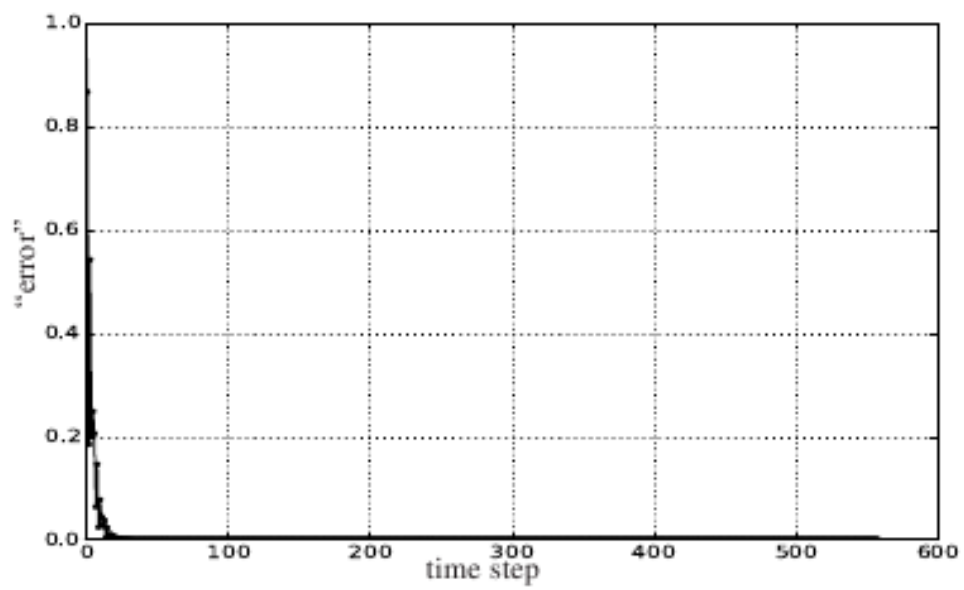

(a)

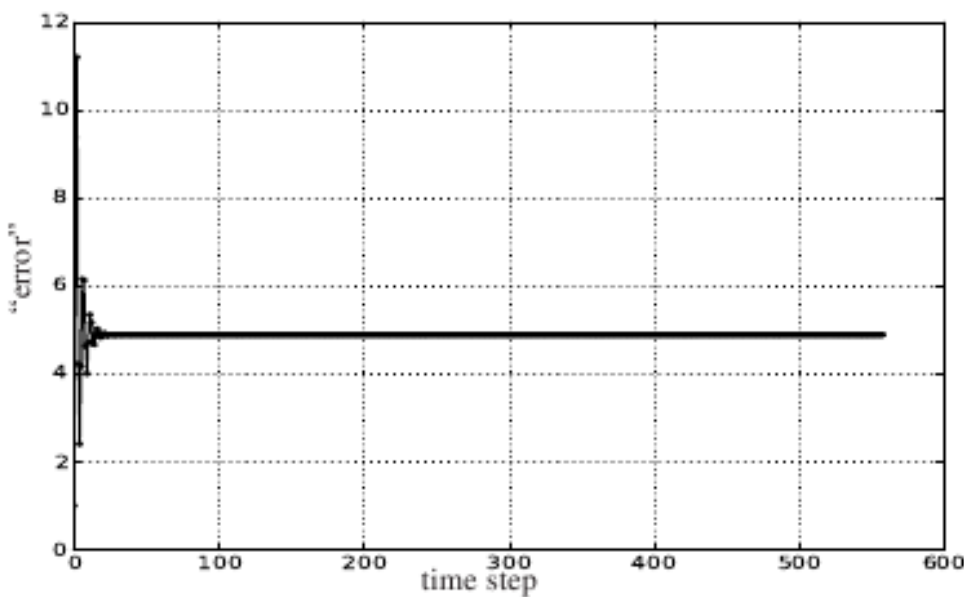

(b)

Figure 6: Evolution of VBEM 'error' for $v=0.0$ and $\beta_{2}=0.8$ (a) velocities (b) tractions.

\section{Conclusions}

A TD-BEM formulation for 3D elastodynamic problems has been proposed and tested. This new formulation is established in terms of velocities and tractions instead of the conventional one, and is based on a power-type reciprocal theorem. This formulation is found to have a better performance regarding stability and its incorporation into existing BEM codes is simple. It only requires the replacement of the displacement fundamental solution by its respective velocity solution as the kernel function. Furthermore, temporal integration of this new kernel is similar to that of the traction kernel, while spatial integrations are 
identical to those of the displacement kernel. This way, we avoid here " "averaging" methods for decreasing instability problems that have hitherto been used, especially in conjunction with 1D formulations. It is also interesting to note that the 2D elastodynamic case can also be formulated in terms of velocitybased quantities and better stability performance is to be expected in this case.

\section{References}

[1] Pierce, A. \& Siebrits, E., Stability analysis of model problems for elastodynamic boundary element discretizations. Numerical Methods for Partial Differential Equations, 12, pp. 585-613, 1996.

[2] Soares, D. \& Mansur, W.J., An efficient stabilized boundary elements formulation for 2D time-domain acoustics and elastodynamics. Computational Mechanics, 40, pp. 355-365, 2007.

[3] Panagiotopoulos, C.G. \& Manolis, G.D., Velocity-based reciprocal theorems in elastodynamics and BIEM implementation issues," Archives of Applied Mechanics, 80(12), pp. 1429-1447, 2010.

[4] Panagiotopoulos, C.G. \& Manolis, G.D., Three-dimensional BEM for Transient Elastodynamics based on Velocity Reciprocal Theorem, Engineering Analysis with Boundary Elements, accepted, to appear in 2011.

[5] Graffi, D., Sul teorema di reciprocita nella dinamica dei corpi elastici. Memoria della Reale Accademia delle Scienze dell'Istituto di Bologna, 10, pp. 103-111, 1946.

[6] Dominguez, J., Boundary Elements in Dynamics, Computational Mechanics Publications \& Elsevier Applied Science, London, 1993.

[7] Polyzos, D., Tsinopoulos, S.V. \& Beskos, D.E., Static and dynamic boundary element analysis in incompressible linear elasticity. European Journal of Mechanics, A/Solids, 17, pp. 515-536, 1998.

[8] Banerjee, P.K, Ahmad, S. \& Manolis, G.D, Transient elastodynamic analysis of 3-D problems by Boundary Element Method, Earthquake Engineering and Structural Dynamics, 14, pp. 933-949, 1986.

[9] Frangi, A., Causal shape functions in the time domain boundary element method, Computational Mechanics 25, pp. 533-541, 2000.

[10] Frangi, A. \& Novati, G., On the numerical stability of time-domain elastodynamic analyses by BEM, Computer Methods in Applied mechanics and Engineering, 173, pp. 403-417, 1999.

[11] Schanz, M., Ruberg, T. \& Kielhorn, L., Time Domain BEM: Numerical aspects of collocation and Galerkin formulations. Recent Advances in Boundary Element Methods, A Volume to Honor Professor Dimitri Beskos, editors: G.D. Manolis \& D. Polyzos, Springer-Verlag, Dordrecht, pp. 415432, 2009.

[12] Pierce, A. \& Siebrits, E., Stability analysis and design of time-stepping schemes for general elastodynamic boundary element models, International Journal for Numerical Methods in Engineering, 40, pp. 319-342, 1997. 